\title{
The Effect of Unloading Positions and Times on Sheet Metal's Stress-Strain Curve
}

\author{
Kunmin Zhao ${ }^{1,2}{ }^{*}$, Wumei Fang1, Baotang Wang1, Daxin Ren ${ }^{1}$ \\ ${ }^{1}$ School of Automotive Engineering, Dalian University of Technology, Dalian, China \\ ${ }^{2}$ Institute of Industrial and Equipment Technology, Hefei University of Technology, Hefei, China \\ Email: *kmzhao@dlut.edu.cn
}

How to cite this paper: Zhao, K.M., Fang, W.M., Wang, B.T. and Ren, D.X. (2018) The Effect of Unloading Positions and Times on Sheet Metal's Stress-Strain Curve. Journal of Minerals and Materials Characterization and Engineering, 6, 408-423. https://doi.org/10.4236/jmmce.2018.63029

Received: March 16, 2018

Accepted: May 19, 2018

Published: May 22, 2018

Copyright $\odot 2018$ by authors and Scientific Research Publishing Inc. This work is licensed under the Creative Commons Attribution International License (CC BY 4.0).

http://creativecommons.org/licenses/by/4.0/

\begin{abstract}
The loading-unloading-reloading process could affect the tensile deformation of metals with the combined function of stress relaxation and work hardening, which has been reported in multiple experiments. Nevertheless, the effects of different unloading positions and unloading times have not been investigated. In this study, unloading-reloading tests on three materials (AL6061, HSLA and Q195) were conducted. The stress exhibits a rapid rise momentarily upon reloading and stabilizes afterward while the post stress-strain curve deviates up or down from the monotonic tensile curve. The ductility is enhanced by the unloading-reloading process in general. Different unloading positions and unloading times have different degrees of influence on the stretching of these metals. The effect of loading conditions on a medium manganese steel was further studied. The functions to modify the post stress-strain relationship after unloading-reloading were established.
\end{abstract}

\section{Keywords}

Sheet Metal, Stretch Forming, Unloading Positions and Times, Elongation, Stress-Strain

\section{Introduction}

Stretching forming as one of the important methods to explore the performance of metal materials has complex plastic forming mechanism. The mechanism of metal plastic deformation during tensile progress is mainly dislocation movement, and the dislocation moving is too difficult to make the elongation change at low temperature [1] [2]. Nevertheless, the mobile dislocation density gradually decreases due to the production of recovery process conducted by slipping or climbing, moreover, the recovery and slipping progress is a combined effect of 
stress relaxation at low temperature showed by the microscopic testing [3]. The loading-unloading cycle progress can affect materials' property during tensile experiments along with stress relaxation and it has great influence on stretch forming. On the one hand, stress relaxation enhances metal ductility markedly confirmed by the increase of elongation [1] [4] [5] [6] [7]. On the other hand, there is a rapid rise in stress momentarily following the stress relaxation because of the time-dependent strain aging and strain hardening [4] [5] [6] [7] [8] or phase transformation [2] [4] [8] [9]. In the course of stress relaxation, the dislocation density decreases gradually and it will increase again upon reloading to reach the previous state. Nevertheless, the dislocation density distributes differently before and after relaxation because of the mutative deformation path. The elongation will increase as a consequence of less density in the critical regions and the variation of flow stress or hardening behavior due to the addition of dislocation density in critical regions owing to the redistribution [2] [3] [4].

Therefore, any factor which could make the material microstructure transformed may attribute to the tensile deformation. First of all, deformation temperature and strain rate must be taken into consideration since they are both involved with influencing the deformation behavior obviously [1] [3] [9]-[14]. The dislocation gliding or climbing is just a kind of manifestation of rate-controlling mechanism essentially [12]. And the high temperature will promote density movement distinctly because of grain boundary sliding [1] [13]. Afterward, aging time and the pre-strain also play an insignificant role in stretch forming [8]. The loading method can be another vital influencing factor with the distinctively different results under three typical loading methods: monotonic, pushed and hold [5] [6] [7] [9] [14]. Furthermore, there are visible stress-strain differences between uniaxial monotonic loading and repeated loading-unloading method of stainless steel 304 [5] [6] [7]. Corresponding constitutive correction functions had been established to reflect the influence of those factors [1] [11].

Most previous studies were focused on the influence of loading method about the metal's tensile property, and the ductility is enhanced under loading-unloading-reloading or incomplete unloading progress. Nevertheless, the effects of unloading positions and times on sheet metal's stress-strain curve have not yet been reported. Therefore, there are two critical questions which is worth to be studied. The first one is whether the unloading positions or unloading times have an effect on sheet metal's stress-strain curve. The second one is whether the correction function depended on unloading positions and times could be established to predict the post stress-strain relationship after reloading provided that there is an apparent effect of those two factors.

In the present study, three materials of AL6061, HSLA and Q195 were studied experimentally to make a confirmation of the effect of unloading and reloading on a stress-strain curve during tensile deformation. Comparative tests were designed to contrast the change of strain-stress curve conducted by unloading position and times. And the total elongation and stress change would be regarded 
as two important metrics. In order to learn the influence of those two factors further and establish constitutive correction functions, several supplementary experiments of medium manganese steel were planned and corresponding correction functions of its stress-strain relationship were established by numerical analysis which would have great importance in the study of enhancing stretching forming of metallic materials.

\section{Experiments}

\subsection{Experimental Description}

The experiments of the effect of the unloading positions and unloading times were tested in AL6061 (an aluminum alloy), HSLA350 (a high strength low alloy steel), Q195 (a structural carbon steel), medium manganese steel (a high strength steel), respectively. The dimensions of the materials shown in Figure 1 were designed according to the national standards and the specimens were designed with rectangular cross-section. The sheet metals were cut into small rectangular pieces using a mechanical shearing machine along the rolling direction (RD) and then finished by wire electric discharge machining (WEDM). And the burrs along the edge were smoothed with sandpaper before the experiments.

All the materials were tested at the same constant rate and other conditions. The tensile tests were performed on a $100 \mathrm{kN}$ servo electric universal testing machine (UTM5105 manufactured by Suns). An electronic extensometer (25 $\mathrm{mm}$ gage length and $50 \%$ strain range) was used to measure the strains.

\subsection{Experimental Scheme}

All the experiments were designed by controlling variables at room temperature and it would merely be affected by unloading position and times with other external conditions being kept the same objectively. The experiment of uniaxial monotonic loading was designed as a reference group while other comparative

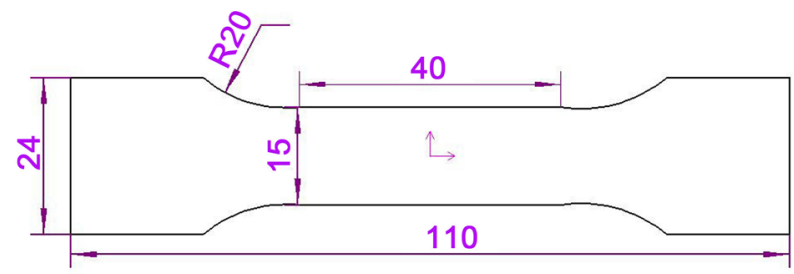

(a)

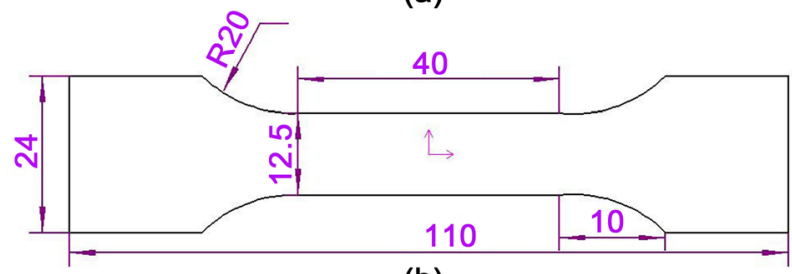

(b)

Figure 1. (a) Dimensions of Q195 and AL6061 tensile test specimen. (b) Dimensions of HSLA350 and medium manganese steel tensile test specimen. 
experiments would vary on the unloading position and times. Several kinds of vehicle light-weight materials were adopted for the experiments, including AL6061, HSLA, Q195 and medium manganese steel. At the first step, a series of experiments were carried on AL6061, HSLA and Q195 to confirm the influence of unloading position and times, and the experiments were designed as Table 1. Firstly, the materials of the reference group were once pulled off directly for getting its total elongation according to the stress-strain curve. For each material, the total elongation of monotonic loading (TEML) was taken into account as the standard for the experiment affected by unloading positions or unloading times, and the percentage of the TEML was defined as the unloading position in all experiments. Secondarily, the unloading-reloading tensile experiment would unload at $50 \%$ of the TEML for once while the twice and three times unloading experiments would unload at $33.3 \%-66.7 \%$ and $25 \%-50 \%-75 \%$ of the TEML in turn with a constant rate, respectively. Furthermore, the deformation would be contrasted by strain-stress curves in the same coordinate system. At the second step, corresponding comparative experiments were designed to come up with the constitutive correction function decided by unloading position and times through data analysis and curve fitting. The material of medium manganese steel was applied and the comparative experiments were designed as Table 2. The monotonic loading test in the comparative experiments is designed as the reference group of the once unloading tests, which include three different groups unloading at $25 \%, 50 \%, 75 \%$ of the TEML. Additionally, there are two groups of twice unloading tests and one group of three times unloading test. The twice

Table 1. Comparative experiments of AL6061, HSLA, Q195.

\begin{tabular}{|c|c|c|}
\hline \multirow{2}{*}{ Reference group } & \multicolumn{2}{|c|}{ Experimental groups } \\
\hline & Total unloading times & Unloading positions in turn \\
\hline \multirow{3}{*}{ Monotonic loading } & 1 & $50 \%$ \\
\hline & 2 & $33.3 \%-66.7 \%$ \\
\hline & 3 & $25 \%-50 \%-75 \%$ \\
\hline
\end{tabular}

Note: The percentage of total elongation of monotonic loading is defined as unloading positions.

Table 2. Comparative experiments of medium manganese steel.

\begin{tabular}{cccc}
\hline \multicolumn{2}{c}{ Experiment groups } & \multicolumn{2}{c}{ Reference groups } \\
\hline Total unloading times & Unloading positions & Total unloading times & Unloading positions \\
\hline & $25 \%$ & 1 & \\
& $50 \%$ & & Monotonic loading \\
& $75 \%$ & 2 & $25 \%$ \\
$25 \%-50 \%$ & 3 & $50 \%$ \\
2 & $50 \%-75 \%$ & 3 & $25 \%-50 \%$ \\
\hline
\end{tabular}

Note: The percentage of total elongation of monotonic loading is defined as unloading positions. 
unloading positions were $25 \%-50 \%$ and $50 \%-75 \%$ of the TEML, respectively, which would be compared to the once unloading group shown in Table 2. As well as, the three times unloading test would unload at $75 \%$ of the TEML once more compared to the twice unloading test that unloaded at $25 \%$ - $50 \%$ of the TEML in turn. Each experiment was repeated for three times to ensure the accuracy of results at room temperature. The influence factors of unloading position and unloading times would be discussed and studied by choosing corresponding comparative experiments separately.

\section{Results and Discussion}

Figure 2 shows the stress-strain curves of loading-unloading experiments of AL6061, HSLA and Q195 with different unloading position and times. And the loading-unloading experiments affected by unloading position and times are compared to monotonic tensile experiment. Partial magnification of stress-strain curve and the total elongation after breaking show the variety clearly. Obviously, unloading progress has a manifest influence on material stretch forming with a rapid rise of stress momentarily, elongation increase and the deviation of curves up or down as a whole after reloading compared to the monotonic tensile curve in general. Moreover, the evident differences are shown by the curves with different unloading position and times by contrasting with each other.

For each material, the maximum stress values of each experiment are extracted from the datum for an intuitive comparison. And the total elongations are obtained from the axis as an indicator of change in formability. What requires noticing especially is that the total elongations of the experiments for Q195 are obtained by curve fitting according to the trend because of the incomplete datum caused by the interruption of extensometer. All of the datum are shown in Table 3.

Comparing with the monotonic loading curve, the stress-strain curve after reloading will deviate upward or downward more or less as a whole while there is a rapid rise of stress momentarily behind the stress achieving to the level before unloading. And the total elongations increase to a distinct degree which is easy to see from the Table 3 . The mobile dislocation density decrease during stress relaxation after loading and it will not increase until reloading to reach the previous state, nevertheless, it will be re-distributed with less density in the critical regions which would delay the necking moment and improve elongation [4]. Meanwhile, the hardening mechanism must be taken into account at low-temperature deformation, as there is complex internal organizational transformation including the producing of slipping, the growing of grain even forming fibrous tissue, and the moving of dislocation. As the dislocation moves, the distribution of dislocation density will be different at different parts or times and produce dislocation plug phenomenon with the quick increase of dislocation density [15]. Work hardening leads to a reduction in the displacement range but the increase of the stresses [16]. Accordingly, it is obvious that the stress has a 


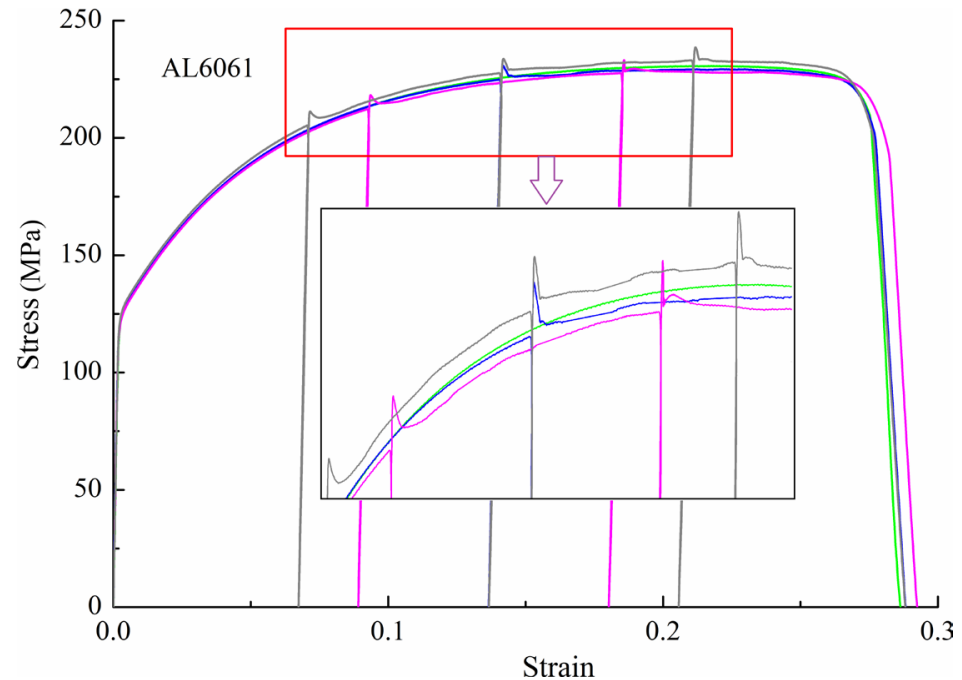

(a)

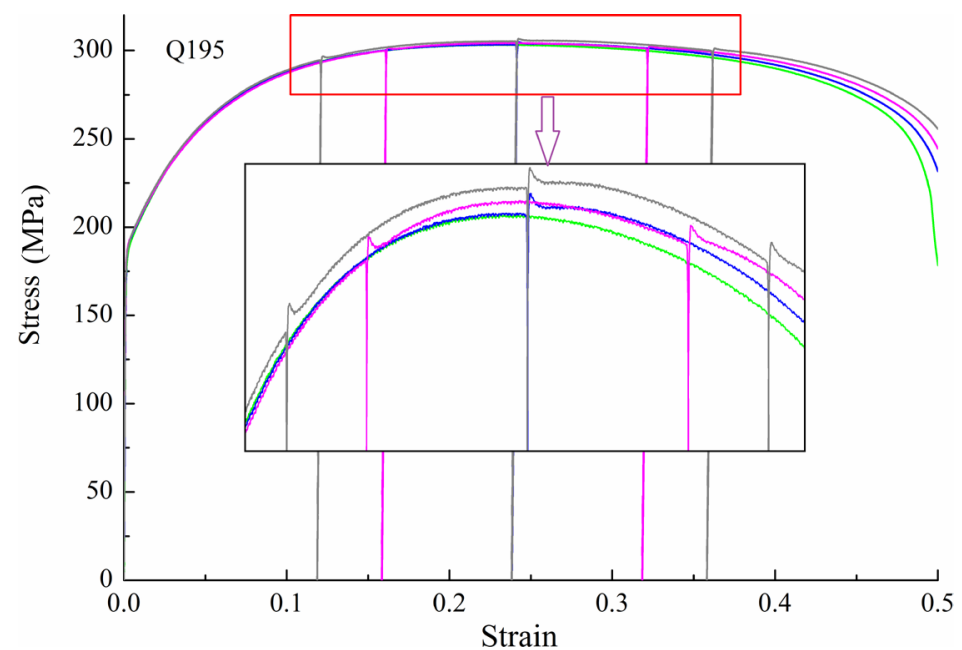

(b)

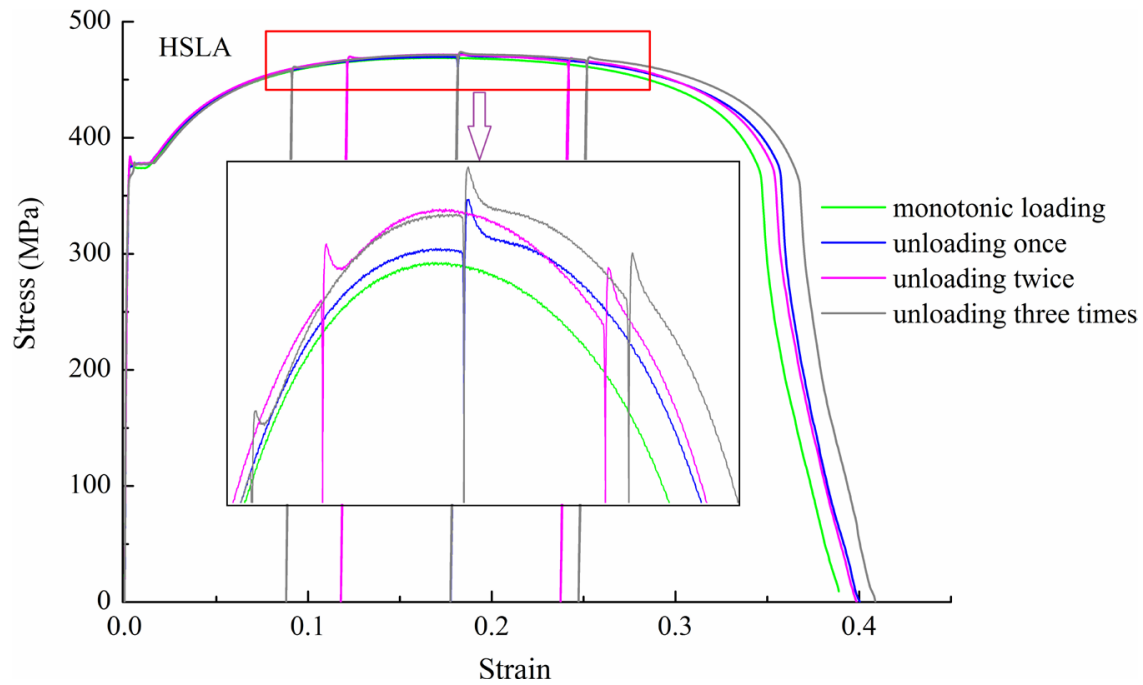

(c)

Figure 2. The stress-strain curves of loading-unloading experiments of AL6061, Q195 and HSLA with different loading position and times compared to the monotonic loading. 
Table 3. The maximum stress values and elongations after breaking of each experiment.

\begin{tabular}{|c|c|c|c|c|c|c|}
\hline Material & Total unloading times & $\sigma_{\max }^{\text {monotonic }}$ & $\sigma_{\max }^{\text {unloading }}$ & $\varepsilon_{\text {monotornic }}$ & $\varepsilon_{\text {unloading }}$ & $\Delta \varepsilon_{\text {ulloading }}(\%)$ \\
\hline \multirow{3}{*}{ AL6061 } & 1 & & 230.840 & & 0.289 & $0.9 \%$ \\
\hline & 2 & 230.753 & 232.687 & 0.286 & 0.292 & $2.2 \%$ \\
\hline & 3 & & 239.378 & & 0.288 & $0.5 \%$ \\
\hline \multirow{3}{*}{ HSLA } & 1 & & 472.864 & & 0.401 & $2.7 \%$ \\
\hline & 2 & 469.607 & 469.663 & 0.390 & 0.399 & $2.3 \%$ \\
\hline & 3 & & 470.935 & & 0.408 & $4.7 \%$ \\
\hline \multirow{3}{*}{ Q195 } & 1 & & 304.797 & & 0.529 & $2.9 \%$ \\
\hline & 2 & 304.705 & 304.216 & 0.514 & 0.546 & $6.2 \%$ \\
\hline & 3 & & 306.707 & & 0.552 & $7.4 \%$ \\
\hline
\end{tabular}

rapid rise momentarily because of the increase of dislocation movement resistance caused by high dislocation density and dislocation tangles [13]. With the help of cross slip, the dislocation could bypass the barrier and lighten work hardening when stress increase to a certain extent [15]. It may be a reasonable explanation that why the curve restores smooth after a rapid rise.

Comparing with the monotonic loading curve, the stress-strain curve of AL6061 unloading at 25\% - 50\% - 75\% of the TEML respectively for three times totally deviates upward, while the curves once unloading at $50 \%$ of the TEML and twice unloading at $33.3 \%-66.7 \%$ of the TEML, in turn, deviate downward slightly. Differently, the curves of HSLA and Q195 deviate upward as a whole after reloading with the one unloading for three times deviates most obviously. At the same time, the total elongation after breaking will increase to a different degree owing to the affection of unloading positions and times. Nevertheless, the maximum elongation change of AL6061 (2.2\%) was conducted by twice unloading at $33.3 \%$ and $66.7 \%$ of the TEML in turn while that was affected by three times unloading at $25 \%-50 \%-75 \%$ of the TEML for Q195 and HSLA, respectively.

As alloy materials with various organizational compositions, so the plastic deformation closely depends on the movement of internal dislocation density, complex changes of grain and solute atoms and other influence factors. The re-precipitation of the strengthening phase and the slight recovery of the dislocation could improve the elongation of AL6061 [17]. There is a series of inclusions in the steel of Q195, while the pearlite content, grain size, ribbon organization and other factors can affect the elongation at the same time [18]. As a steel plate with biphasic or three-phase microstructure, HSLA exhibits a strong work hardening behavior with the increase of stress [19]. No matter how different the microstructure transforms during tensile deformation in a micro perspective, the visible effect of unloading position and unloading times is confirmed by three different materials obviously. Furthermore, corresponding research about this effect will be studied more in-depth in a macro perspective. 
In order to reveal the tendency of those two factors affected on sheet metal's stress-strain curve in the course of tensile forming and establish correction function to predict the stress-strain relationship more accurately contributed by unloading positions and times, therefore, the comparative experiments are designed with medium manganese steel shown in Table 2 and their stress-strain curves are shown in Figure 3. In Figure 3(a), there are three groups of once unloading

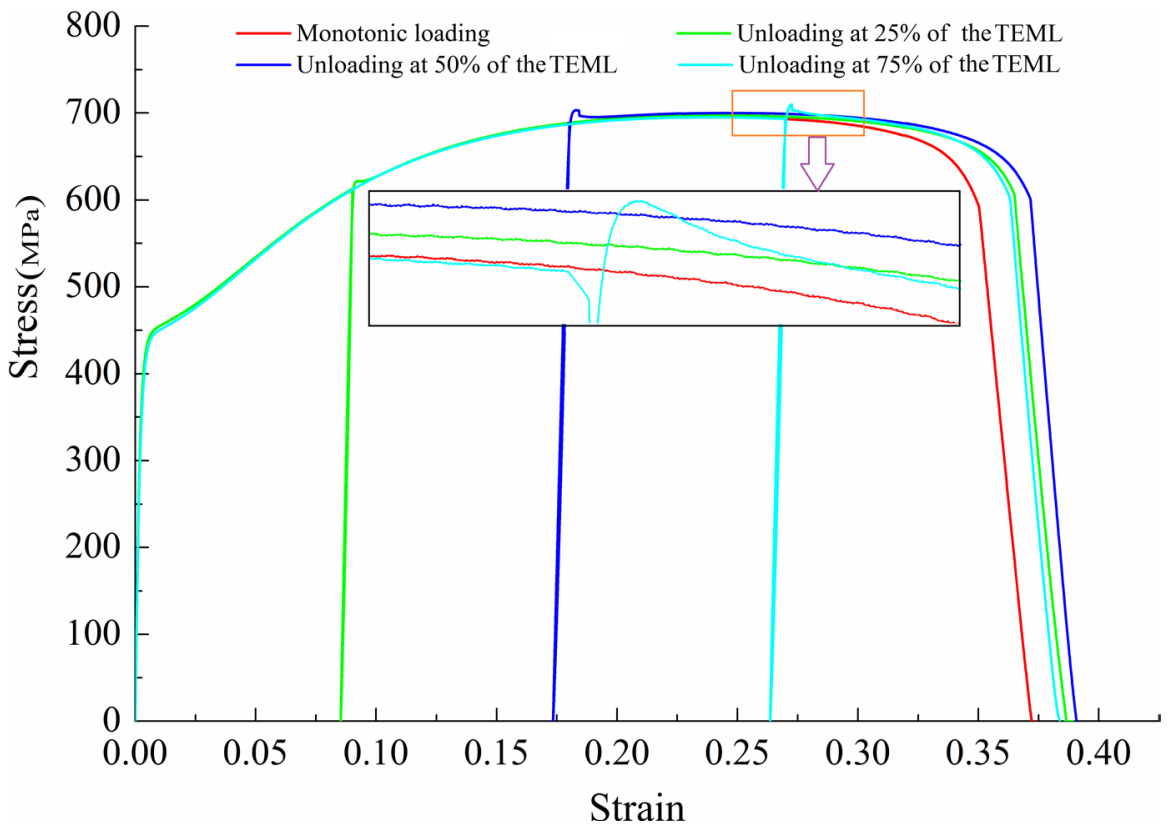

(a)

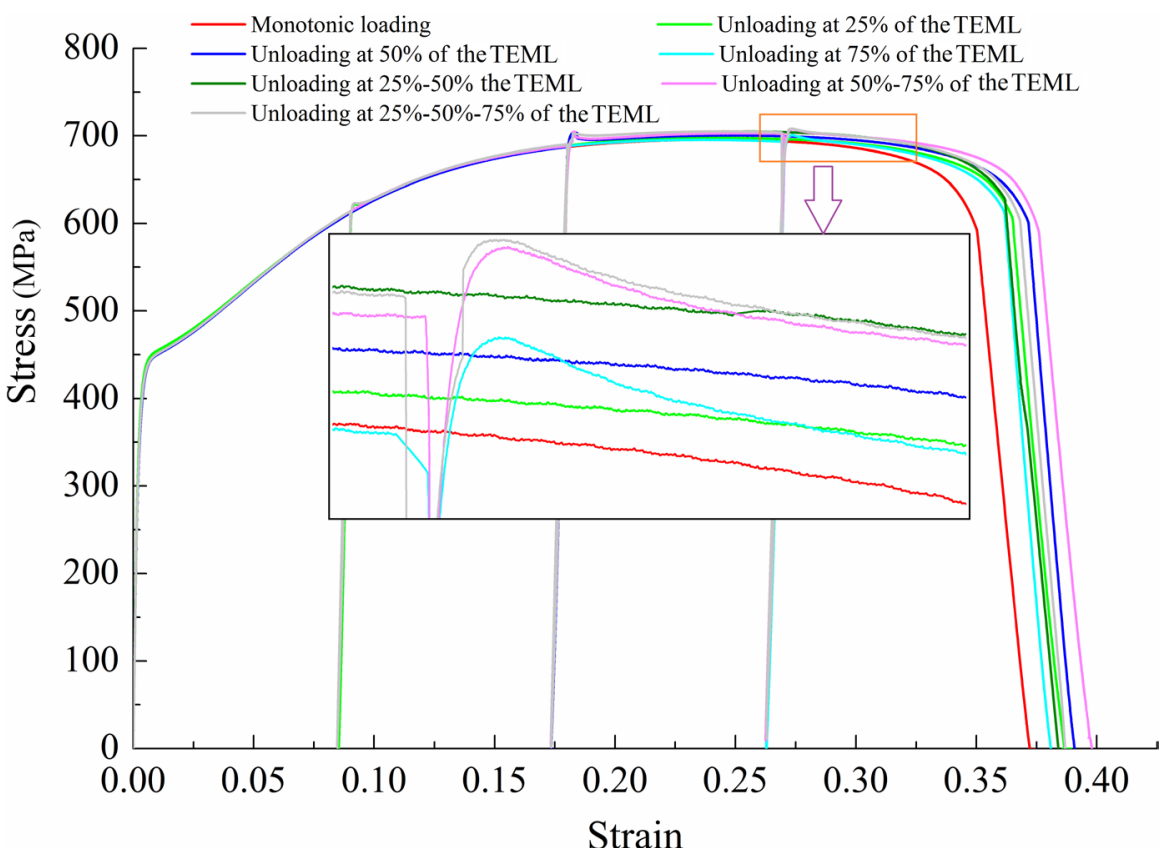

(b)

Figure 3. The strain-stress curves of medium manganese steel affected by different unloading positions and unloading times. (a) Unloading once at different positions. (b) Comparative experiments of different unloading times at $75 \%$ of the TEML. 
experiments compared to the monotonic loading experiment with different unloading positions and the effect on the stress-strain curve is shown by the partial magnification. Moreover, all the stress-strain curves of the comparative experiments are shown in Figure 3(b). Similarly, the effect of different unloading times on stress-strain curve when unloading at $75 \%$ of the TEML by comparing with the reference group is shown by the partial magnification. And the total elongation of each experiment and its changed value compared to the monotonic loading or the reference group is shown in Table 4.

The evident change of medium manganese steel's stress-strain curve affected by different unloading positions is shown in Figure 3(a). The curve unloading at $50 \%$ of the TEML converts most obviously in comparison with the one unloading at $25 \%$ or $75 \%$ of the TEML, which the total elongation increases $5.05 \%$ compared to the monotonic loading and its stress-strain curve deviates upward most obviously. Meanwhile, the effect of different unloading times at $75 \%$ of the TEML by the comparison of each comparative experiment is shown in Figure 3 (b). In order to show the comparison evidently, all the experiments are numbered. By the contrast of group 5 with group 2, group 6 with group 3, group 7 with group 5 respectively, which will show the difference of different unloading times (the $1^{\text {st }}$, the $2^{\text {nd }}$, the $3^{\text {rd }}$ ) at $75 \%$ of the TEML. And the first time unloading at $75 \%$ of the TEML has a maximum increase $(2.5 \%)$ of total elongation where the increase of total elongation reduced while the unloading times increase. And the deviation degree of the stress-strain curve of unloading at $75 \%$ of the TEML increases in company with the increased unloading times while the $2^{\text {nd }}$ and the $3^{\text {th }}$ have a nearby deviation. Especially, when the second time unloads at $50 \%$ of the TEML, the group 5 has a reduction on total elongation compared to the group 2 which just unloads at $25 \%$ of the TEML for the first time. Generally speaking, the different unloading positions and times exert a different effect on stress-strain curve evidently.

Table 4. The total elongation and its change value of medium manganese steel affected by different unloading positions and times.

\begin{tabular}{ccccccc}
\hline $\begin{array}{c}\text { Experiment } \\
\text { group } \\
\text { number }\end{array}$ & $\begin{array}{c}\text { Total } \\
\text { unloading } \\
\text { times }\end{array}$ & $\begin{array}{c}\text { Unloading } \\
\text { position (\%) } \\
\text { in turn }\end{array}$ & $\varepsilon_{\text {max }}$ & $\begin{array}{c}\Delta \varepsilon(\%) \\
\text { Compared to } \\
\text { TEML) }\end{array}$ & $\begin{array}{c}\Delta \varepsilon(\%) \text { (Compared to } \\
\text { reference group) }\end{array}$ \\
\hline 1 & & Monotonic loading & 0.3723 & -- & -- & -- \\
Rroup & $\Delta \varepsilon(\%)$ \\
2 & 1 & $25 \%$ & 0.3868 & $3.89 \%$ & 1 & $3.89 \%$ \\
3 & 1 & $50 \%$ & 0.3911 & $5.05 \%$ & 1 & $5.05 \%$ \\
5 & 1 & $75 \%$ & 0.3816 & $2.50 \%$ & 1 & $2.50 \%$ \\
6 & 2 & $25 \%-50 \%$ & 0.3845 & $3.28 \%$ & 2 & $-0.6 \%$ \\
7 & 2 & $50 \%-75 \%$ & 0.3982 & $6.96 \%$ & 3 & $1.82 \%$ \\
\hline & 3 & $25 \%-50 \%-75 \%$ & 0.3873 & $4.03 \%$ & 5 & $0.73 \%$ \\
\hline
\end{tabular}


Not only high-density dislocation and stacking reaction, but also phase transformation with carbide formation and martensite growth following the increase of strain are observed from medium manganese steel by transmission electron microscopic. Those would hinder or even stop the expansion of the crack. The work hardening is a combined effect of density dislocation, stack fault, the second phase particles precipitated and hardened, and strain induced martensite [20]. The similar research is reported that the retained austenite of medium manganese steel could relax the concentrated stress and delay the formation of cracks, moreover, the first martensitic transformation would occur in the largest deformation parts and enhance the strength while promoting the deformation to a smaller deformation area. That would delay the formation of necking and exert a major part in enhancing plasticization [2]. All of those closely relevant to strain during deformation as it is dynamic progress which will be affected by unloading position and times easily. As the mobile dislocation density is time-dependence [21]. It is not hard to know that dislocation density and its distribution, martensitic transformation and the movement and distribution of other organizational elements are different at different strain moment. As a consequence, the different unloading position would change the state of those before unloading at a different degree that would increase the dislocation movement resistance and delay fracture, which reflects the increased of stress and total elongation macroscopically. Similarly, different unloading times at the same position would affect the deformation progress differently. Therefore, the corresponding correction function should be established to reveal the influence and forming the forecasting model to predict the relationship of stress-strain with different unloading position and times in the plastic stage.

The stress-strain relationship in the plastic stage is assumed as $f(\varepsilon)$ and the correction function related to different unloading positions and times is $y(P, N)$, in which $P$ and $N$ are on the behalf of unloading positions and unloading times, respectively. Thereafter, the relationship between stress and strain will gratify $f^{\prime}(\varepsilon, P, N)$ after unloading at different positions for different times. Afterward, the modified function relationship is shown as Equation (1).

$$
f^{\prime}(\varepsilon, P, N)=f(\varepsilon) * y(P, N) .
$$

The stress-strain curves of the experiment groups are compared to the reference groups' curves accordingly in the same coordinate system, and the post stress-strain curves after the last unloading position of the plastic stage are extracted. Moreover, the proportional values of those two stress-strain curves of each comparative group after the last unloading position of experiment group but before the necking moment are gained. Consequently, there are proportional curves of the comparative experiments and the corresponding fitting curves are shown in Figure 4.

The best fitting function is exponential and the fitting curves match the form of Equation (2) extremely well. 


$$
y(\varepsilon)=y_{0}+A^{*} \exp \left(-\frac{\varepsilon}{t}\right) .
$$

The fitting results of the proportional curve of each comparative experiment are shown in Table 5. It indicates that each parameter is a variable associated with the unloading positions and unloading times. Thus, the relationships between each parameter $\left(y_{0}, A, t\right)$ and the unloading positions $(P)$ and times $(N)$ will be discussed. Distinctly, it's changeable of each parameter with the change of $N$ and $P$ and the relationship is decided by those two factors at the same time. To take those two factors into consideration together, the quadratic polynomial function will be adapted for fitting the relationship of each parameter with $N$ and $P$. The fitting results are shown in Figure 5.

As the pictures show above, the form of a binary quadratic polynomial is applied to fit the datum. Subsequently, the correction functions are established

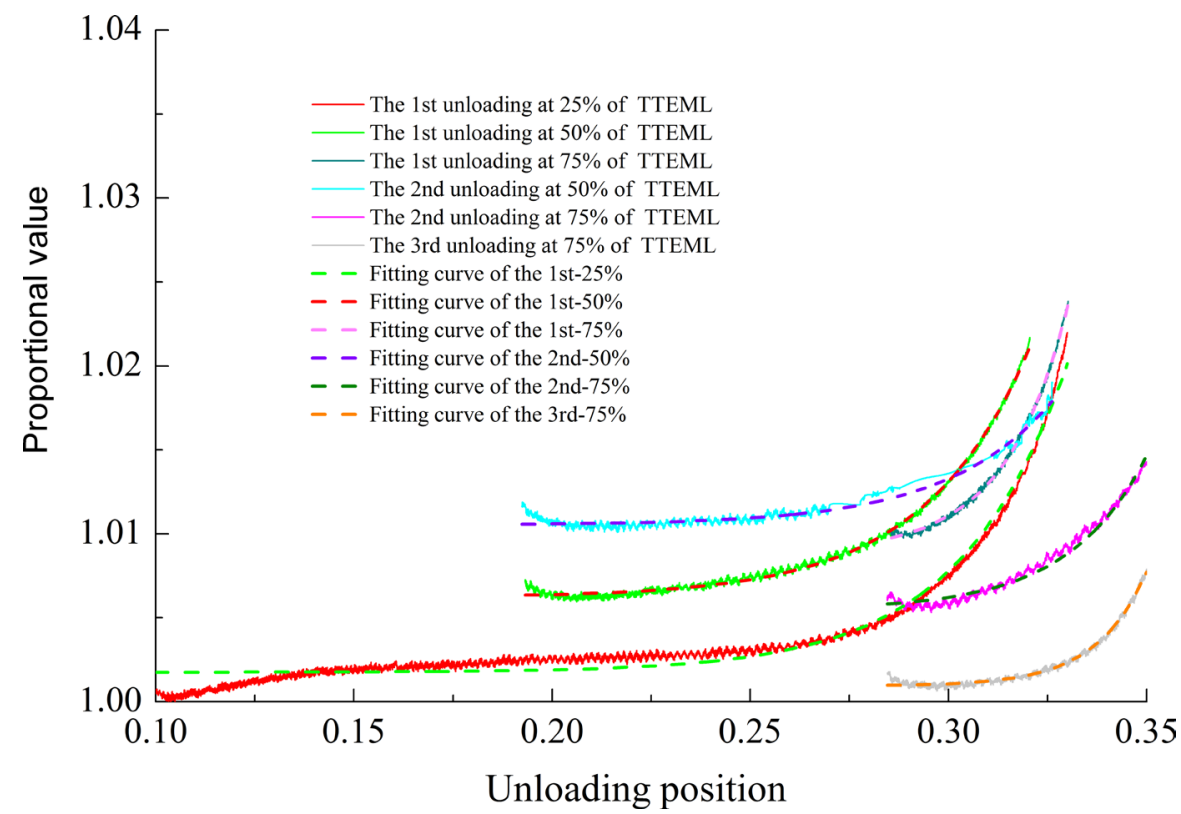

Figure 4. The proportional curve and its fitting curve of stress-strain curve with different unloading positions and times of each comparative experiment respectively.

Table 5. The fitting results of the proportional curve of each comparative experiment.

\begin{tabular}{|c|c|c|c|c|c|c|c|}
\hline \multicolumn{2}{|c|}{ The comparative experiments } & \multicolumn{2}{|c|}{ Influencing factors } & \multicolumn{3}{|c|}{ Parameters of the correction function } & \multirow[b]{2}{*}{ R-Square } \\
\hline $\begin{array}{c}\text { Experiment } \\
\text { group }\end{array}$ & $\begin{array}{c}\text { Contrasted } \\
\text { group }\end{array}$ & $\mathrm{N}$ & $\mathrm{P}$ & $\mathrm{y}_{0}$ & A & $\mathrm{t}$ & \\
\hline 2 & 1 & 1 & $25 \%$ & 1.00175 & $8.70841 \mathrm{E}-8$ & -0.02692 & 0.96734 \\
\hline 3 & 1 & 1 & $50 \%$ & 1.00622 & $8.86433 \mathrm{E}-8$ & -0.02664 & 0.99706 \\
\hline 4 & 1 & 1 & $75 \%$ & 1.00886 & $1.35951 \mathrm{E}-13$ & -0.01587 & 0.99870 \\
\hline 5 & 2 & 2 & $50 \%$ & 1.01052 & $3.53175 \mathrm{E}-8$ & -0.02663 & 0.97508 \\
\hline 6 & 3 & 2 & $75 \%$ & 1.00549 & $1.8009 \mathrm{E}-10$ & -0.01973 & 0.99715 \\
\hline 7 & 5 & 3 & $75 \%$ & 1.00094 & $7.6093 \mathrm{E}-15$ & -0.01272 & 0.99653 \\
\hline
\end{tabular}




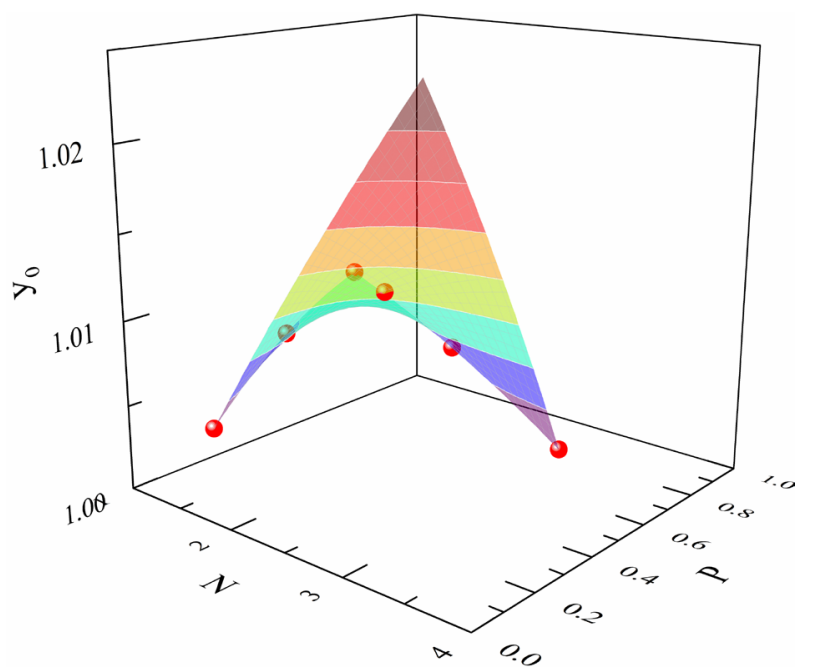

(a)

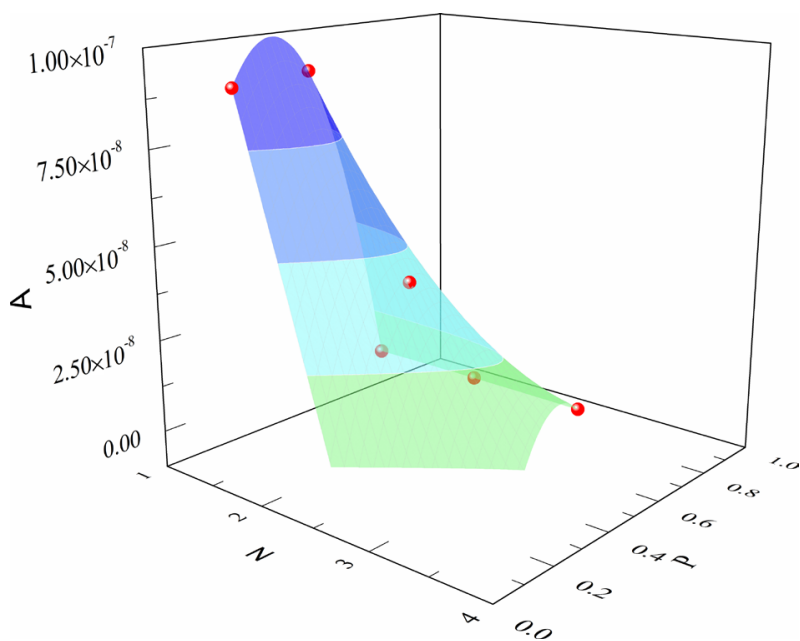

(b)

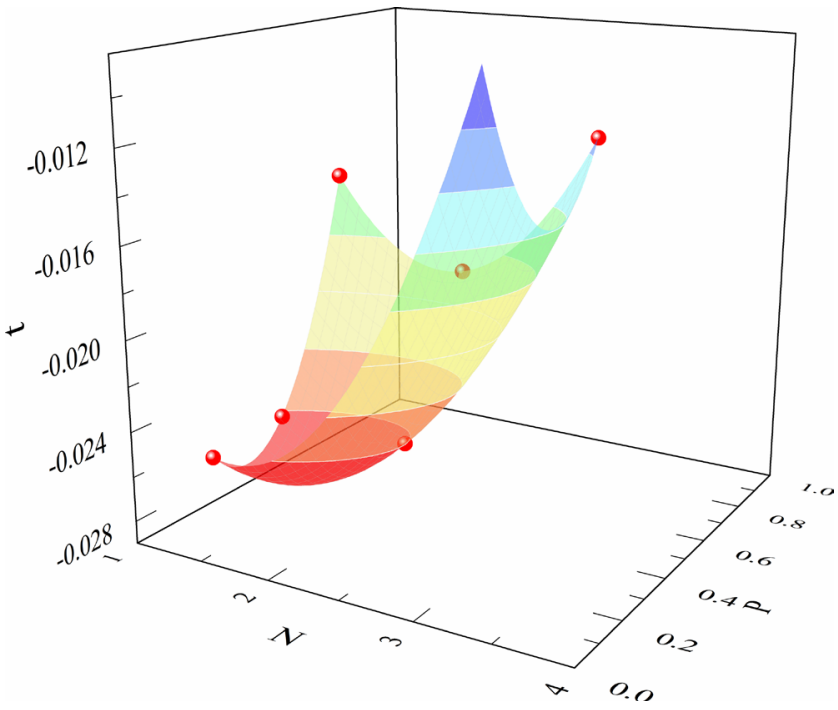

(c)

Figure 5. The fitting results of the relationship related to the unloading positions and times of each parameter. 
which is up to unloading positions $(\mathrm{P})$ and unloading times $(\mathrm{N})$ to decide. So that the post stress-strain relationship after reloading could be predicted with the correction functions below.

$$
\begin{gathered}
A=1.553 * 10^{-7}-1.598 * 10^{-7} N+3.334 * 10^{-7} P-1.8 * 10^{-10} N^{2} \\
+2.14 * 10^{-7} P N-7.216 * 10^{-7} P^{2} \\
y_{0}=0.9746+0.02141 N+0.05954 P-0.00059 N^{2}-0.03068 P N-0.01464 P^{2} \\
t=-0.01359-0.008555 N-0.04634 P+0.005436 N^{2}-0.01548 P N+0.08392 P^{2}
\end{gathered}
$$

As each parameter of the proportional function which is shown in Equation (2), depending on the unloading positions and times, so substituting the above equation into the proportional function that the correction function of $y(P, N)$ is established perfectly. With the revised model, the post stress-strain curve after unloading-reloading at any positions for any times will be predicted. In order to test and verify the accuracy of this correction function, the prediction curve of unloading at $25 \%-50 \%$ of the TEML will be tested and compared to the actual experiment curve. After the first unloading position of $25 \%$ of the TEML, that $\mathrm{N}$ and $\mathrm{P}$ will be assigned 1 and 0.25 , respectively, while 2 and 0.5 are assigned in the post stress-strain curve after unloading at $50 \%$ of the TEML for the second time, respectively. The contrasted result is shown in Figure 6. The prediction curve obtained by the correction function coincides with the experiment's curve trend well.

Precise stress-strain relationship affected by different unloading position and times of medium manganese steel will be gained with the help of the correction function. It is a significant improvement for the study about this material. The accurate function relationship between stress and strain after unloading-reloading at any position or for any times at plastic stage will be conducted by the correction

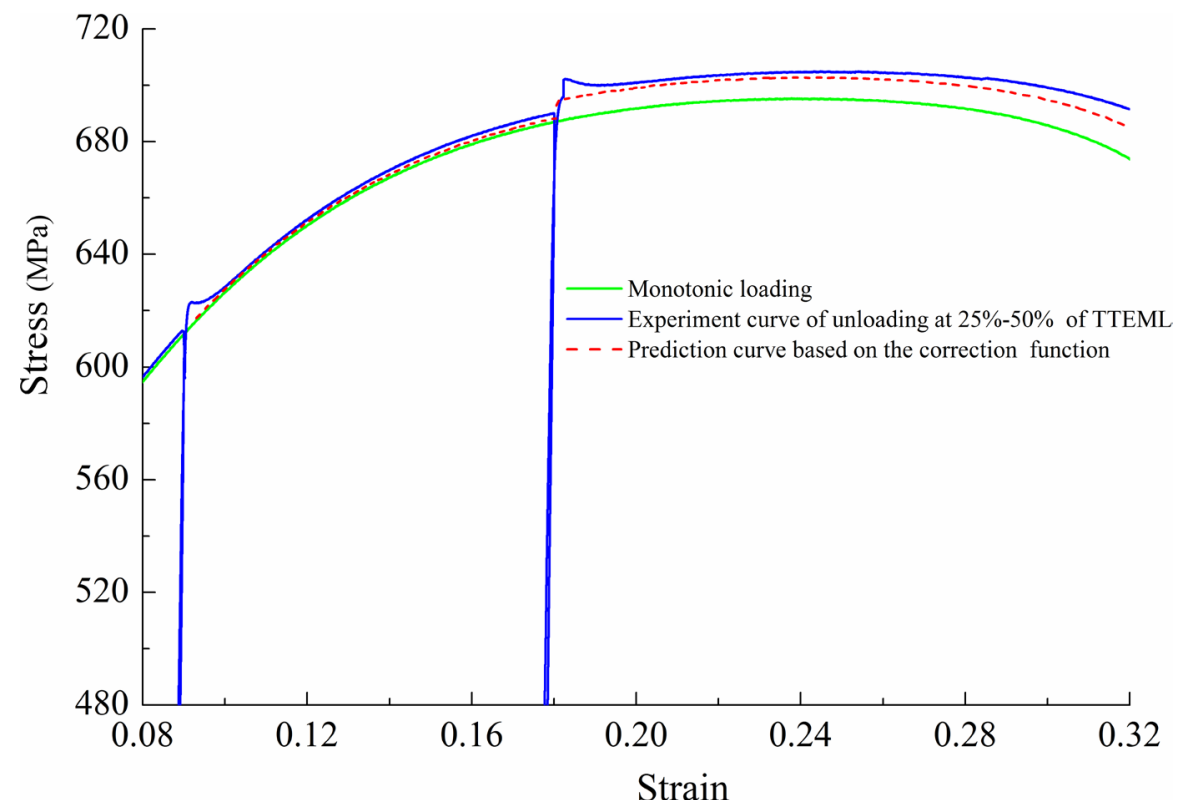

Figure 6. The validation result of the curve unloading at $25 \%-50 \%$ of the TEML 
function. Furthermore, the method can also be used for any material with unloading-reloading progress during the tensile experiment to gain precise datum, which will be a great contribution to the relevant research.

\section{Conclusions}

The effect of unloading and reloading on sheet metals' stress-strain curves is confirmed by different materials (AL6061, HSLA and Q195) and corresponding correction functions for medium manganese steel are established to predict the influence of unloading position and times on its stress-strain curve during tensile forming.

1) Compared to monotonic loading, the stress rises rapidly upon reloading and then becomes smooth with the post stress-strain curve deviated from the monotonic curve either upward or downward while the ductility is always enhanced. Three times unloading impacts Q195 and HSLA the most: the stress-strain curves both deviate upward and the total elongations increase by 7.4\% and 4.7\% (percentage change), respectively. For AL6061, twice unloading affects the total elongation the most with a $2.2 \%$ increase; the stress-strain curves of once and twice unloading deviate downward from the monotonic curve, but the one of three times unloading deviates upward.

2) Different unloading positions and unloading times affect the medium manganese steel's stress-strain curve differently. The total elongation of once unloading at $50 \%$ of the TEML increases $5.05 \%$ compared to monotonic loading, which is greater than the total elongation of once unloading at $25 \%$ or $75 \%$ of the TEML. Moreover, the stress-strain curve after unloading at the same position of $75 \%$ of the TEML for different times has a different variation by the comparison and the first time unloading at $75 \%$ of the TEML has a maximum increase $(2.5 \%)$ of total elongation where the increase of total elongation reduced while the unloading times increase. And the deviation degree of the stress-strain curve of unloading at $75 \%$ of the TEML increases in company with the increased unloading times while the 2 nd one and the 3rd one have a nearby deviation. Especially, the once unloading at $50 \%$ of the TEML for the second time and unloading at $25 \%$ of the TEML for the first time has a reduction of total elongation compared to the one single unloading at $25 \%$ of the TEML.

3) Corresponding correction functions are established to predict the influence of loading conditions on medium manganese steel in the course of tensile forming. The proportional curves of each group of comparative experiment which relates to unloading position and unloading times are extracted and the correction functions are established based on the fitting results. So the post stress-strain relationship after unloading-reloading at any position or for any times will be predicted on the basis of the monotonic loading curve with the help of the correction functions.

\section{Acknowledgements}

This research was supported by the National Natural Science Foundation of 
China (51775160, 11472072). Authors would like to thank Dr. Jilei Hu of Huazhong University of Science and Technology for his good suggestions to the article. Also, authors are appreciated to Mr. Liming Wang of Dalian University of Technology who provided parts of the experimental datum for this study.

\section{References}

[1] Wei, W. and Liu, C.Z. (2011) Research on Constitutive Relationship and Deformation Behavior of 2198 AL-Li Alloy (Chinese). Master. Thesis, Shenyang Aerospace University, Shenyang.

[2] Dong, R. (2015) Microstructure Evolution and Transformation Induced Plasticity Behavior of Medium Manganese Steel (Chinese). Ph.D. Thesis, University of Science and Technology Beijing, Beijing.

[3] Xiao, L. and Bai, J.L. (1998) Stress Relaxation Properties and Microscopic Deformation Structure of H68 and QSn6.5-0.1 Copper Alloys at 353 K. Materials Science and Engineering A, 244, 250-256. https://doi.org/10.1016/S0921-5093(97)00688-6

[4] Hariharan, K., Majidi, O., Kim, C., Lee, M.G. and Barlat, F. (2013) Stress Relaxation and Its Effect on Tensile Deformation of Steels. Materials \& Design, 52, 284-288. https://doi.org/10.1016/j.matdes.2013.05.088

[5] Cullen, G.W. and Korkolis, Y.P. (2013) Ductility Enhancement in Pulsed Uniaxial Tension of 304 Stainless Steel: Experiments and Analysis. AIP Conference Proceedings, American, May 2013, 725. https://doi.org/10.1063/1.4806902

[6] Cullen, G.W. and Korkolis, Y.P. (2010) Ductility Enhancement in Pulsed Uniaxial Tension of Stainless Steel 304. International Manufacturing Science \& Engineering, Pennsylvania, 12-15 October 2010, 653-659. https://doi.org/10.1115/MSEC2010-34160

[7] Cullen, G.W. and Korkolis, Y.P. (2013) Ductility of 304 Stainless Steel under Pulsed Uniaxial Loading. International Journal of Solids and Structures, 50, 1621-1633. https://doi.org/10.1016/j.ijsolstr.2013.01.020

[8] Hannula, S.P., Korhonen, M.A. and Li, C.Y. (1986) Strain Aging and Load Relaxation Behavior of Type 316 Stainless Steel at Room Temperature. Metallurgical \& Materials Transactions A, 17, 1757-1767. https://doi.org/10.1007/BF02817274

[9] Xu, Y., Zhang, S.H., Chen, M. and Song, H.W. (2013) Effect of Loading Method on Mechanical Properties and Martensitic Transformation of Austenitic Stainless Steel. Journal of Metals, 49, 775-782. (In Chinese)

[10] Li, X., Li, L.X. and Ye, T. (2014) Constitutive Model Optimization of 6013 Aluminum Alloy Based on Material Parameter Correction. Chinese Journal of Nonferrous Metals, 6, 1393-1400. (In Chinese)

[11] Krempl, E. (2001) Relaxation Behavior and Modeling. International Journal of Plasticity, 17, 1419-1436. https://doi.org/10.1016/S0749-6419(00)00092-9

[12] Kim, J.H., Byun, T.S. and Hoelzer, D.T. (2012) Stress Relaxation Behavior of Nanocluster-Strengthened Ferritic Alloy at High Temperatures. Journal of Nuclear Materials, 425, 147-155. https://doi.org/10.1016/j.jnucmat.2011.06.040

[13] Gupta, I. and Li, J.C.M. (1970) Stress Relaxation, Internal Stress, and Work Hardening in Some Bcc Metals and Alloys. Metallurgical Transactions, 1, 2323-2330. https://doi.org/10.1007/BF02643451

[14] Majidi, O., De Cooman, B.C., Barlat, F., Lee, M.G. and Korkolis, Y.P. (2016) Thermomechanical Response of A TWIP Steel during Monotonic and Non-Monotonic 
Uniaxial Loading. Materials Science and Engineering A, 674, 276-285. https://doi.org/10.1016/j.msea.2016.08.002

[15] Li, R. (2013) Metallic Plastic Forming Principle: Theory, Analysis, and Inference. 2th Edition, Machinery Industry Press, Beijing.

[16] Ellyin, F. and Fakinlede, C.O.A. (1987) A Dislocation Model for Work Hardening Material and Cyclic J-Integral. International Journal of Fracture, 33, 95-110.

[17] Li, H., Mao, Q., Xiu, Z., et al. (2014) Effect of Pre-Aging + Cold Rolling Deformation + Rejuvenation on Microstructure and Mechanical Properties of 6061 Aluminum Alloy. Journal of Metals, 10, 1244-1252. (In Chinese)

[18] Zhao, Q., Zhou, G. and Zhao, J. (2005) Analysis on the Reason of Low Elongation of the Strip. Physical and Chemical Testing-Physics Volume, 41, 283-285. (In Chinese)

[19] Hulka, K. and Shi, X. (2005) Application of Modern Multi-Phase Steel in the Automobile Industry. Anshan Iron and Steel Technology, 5, 58-62. (In Chinese)

[20] Xie, J., Zhu, Y., Li, Q. and He, Z. (1999) In Situ Tensile Observation of Manganese Steel by Transmission Electron Microscope. Journal of Electron Microscopy, 18, 529-535. (In Chinese)

[21] Meyers, M.A., Guimarães, J.R.C. and Avillez, R.R. (1979) On Stress-Relaxation Experiments and Their Significance under Strain-Aging Conditions. Metallurgical Transactions A, 10, 33-40. https://doi.org/10.1007/BF02686403 